\title{
Cylindrical Three-Hole Pressure Probe Calibration for Large Angular Range
}

\author{
Argüelles Díaz, K.M.; Fernández Oro, J.M.; \\ Blanco Marigorta, E.
}

\author{
Universidad de Oviedo, Área de Mecánica de Fluidos. \\ Campus de Viesques, 33271, Gijón (Asturias), Spain. \\ arguelleskatia@uniovi.es
}

\begin{abstract}
This paper presents a brand new method to calibrate a Cylindrical Three-Hole Probe (CTHP) in a "non-nulling" operating mode. This calibration method employs the definition of a new normalization factor used in the calibration coefficients of the probe. By means of this new normalization, singular points are preserved to appear in the calibration coefficients. In addition, the angular range attainable when using this new calibration is increased in 80 degrees with respect to the typical angular ranges of traditional calibrations. To validate this study, an uncertainty analysis of the probe using this new method is carried out, resulting in significantly lower uncertainties for the whole angular range of the probe. Complementarily, the influence of the angular distance between the holes of the probe on both angular ranges and maximum uncertainties is also performed in the paper. From this analysis, it is established that the optimal angular separation for the holes must be chosen to be between 50 and 60 degrees. Finally, to illustrate the usefulness of this procedure, a set of experimental measurements were conducted downstream of the rotor of an axial turbomachine, using the new normalization factor in a CTHP with an angular distance of 60 degrees between the holes. The results revealed that the complex, unsteady flow within the blade passages can be perfectly captured, including those regions with high variations in the flow angle, like the shear layers of the wake fluid or both casing and hub boundary layers. In summary, this newly developed calibration method enables to measure large variations of the flow angle, in particular, up to 140 degrees.
\end{abstract}

\section{INTRODUCTION}

Multihole pressure probes present notable advantages with respect to hot-wire probes and laser doppler velocimetry (LDV) systems, since both pressure and velocity magnitudes are simultaneously available when measuring pressure differentials. Introducing miniature sensors with high frequency response, it is possible to measure unsteady flows, wakes behind moving elements and even turbulence.

In the case of certain unsteady flows, like those encountered within turbomachinery environments, the magnitude and direction of the velocity fields may present important variations over their integral time scales. The typical angular range of three- and five-hole probes is usually around $\pm 30^{\circ}$, which is wide enough for a large number of applications. However, while conducting an experimental survey to measure the flow field in an axial turbomachine with a CTHP, it was found that the angular range of the probe was inadequate. At a first glance, it was thought that the angular distance between the holes, 45 degrees, was the key parameter limiting the angular range. Based on previous experiences and knowledge in the design of hot-wire probes ([1]), the authors decided to built a new CTHP with a larger distance between the holes, i.e. 60 degrees. It would be expectable that a larger distance between the holes would offer a wider angular range of the probe. Surprisingly, the results that were obtained after the calibration of the modified probe showed that no improvement in the angular range was achieved increasing the holes angular separation. This fact is illustrated in figure 1 , representing the experimental angular coefficient for both probes: the white-dotted line for an angular distance of 60 degrees and the graydotted line for 45 degrees. Note that the angular range is exactly the same in both cases: $\pm 30^{\circ}$.

As a consequence of this astonishing conclusion, it was initiated a new investigation about the behavior of the calibration coefficients in multihole pressure probes. The investigation led to the establishment of a calibration procedure based on the definition of a new normalization factor, which is responsible for an outstanding increment of the angular range of the probes: up to 70 degrees.

In this paper, an analysis of the calibration coefficients that are obtained after introducing the new normalization factor is carried out. It will be shown that 
the characteristics of the calibration can be justified from the pressure distribution around a cylinder. In addition, the uncertainty levels that are derived from this new normalization are presented. To complete the analysis, the influence of the angular distance between the CTHP holes -denoted as " $\delta$ " throughout the paper- is also studied in detail.

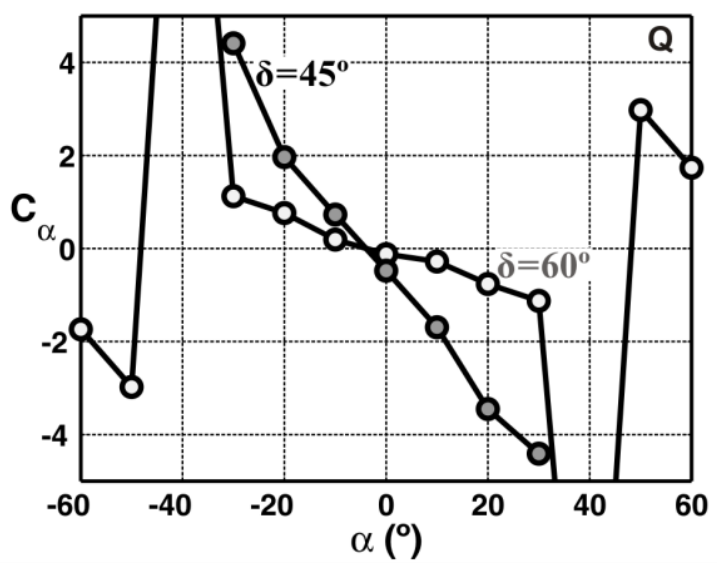

\section{Figure 1. Experimental angular coefficient for a CHTP with angular distance of $60^{\circ}$ and $45^{\circ}$.}

Finally, the calibration coefficients obtained for a CTHP with an angular distance of 60 degrees between the holes are documented. In addition, the probe was developed and used in a study of the flow downstream of the rotor of an axial turbomachine.

\section{BACKGROUND}

Among all the types of pressure probes that have been developed to obtain two-dimensional velocities in fluid flows, the most widely used are those designed with three holes (THP). Obviously, these probes are composed of three pressure transducers placed in a holder. The holder can adopt different geometries, being the most popular the wedge-shaped and the cylindricalshaped probes. In this study we are just considering three-hole pressure probes mounted on a cylindrical arrangement. References [2] and [3] present a complete review of the different topologies of probes including the main operative characteristics.

The pressure distribution over the face of a multihole probe depends on the angle that is defined between the incidence of the flow and the probe axis. In order to determine the direction (two-dimensional) and the magnitude of the flow, the pressure on the probe face must be necessary measured at least in three taps. The first one must be placed on the direction of the measurements while the others are equally spaced towards both sides of the probe. A typical sketch with this configuration is shown in Fig. 2. The pressure that is measured in the central port provides the stagnation conditions when the flow is aligned with the hole. The difference between the pressures felt in the lateral ports is related to the flow incidence angle according to an appropiate calibration method.

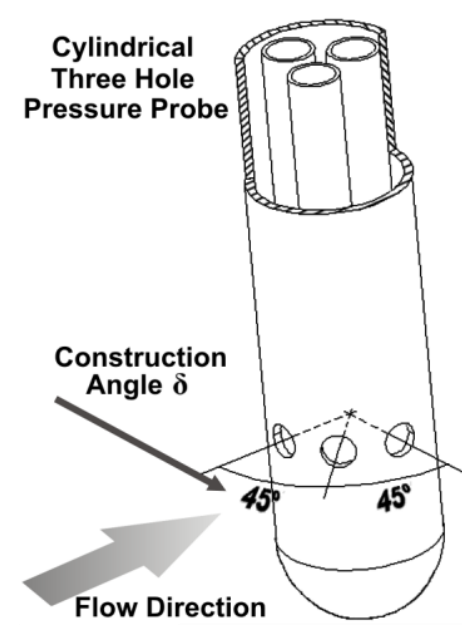

Figure 2. Sketch of a CTHP.

By definition, the pressure probes constitute a typical intrusive method for measuring velocity fields. Since it is desirable to guarantee a reduced blockage of the probe in the freestream, the front face of the holder must be as small as possible, as long as the structural integrity of the probe is not compromised. Besides, all the flow parameters must be measured with the lowest distortion over the flow field. Under these constraints, the probe geometry must be a slender body, with a characteristic radius considerably smaller than the probe length. Though it would be expectable that a holder aligned streamwise should provide optimal results, a circular crossed-section probe presents less negative effects on the angle of attack of the ports when the flow is not normal to the probe ([4]).

Pressure probes present two different operating modes. The simplest one, in terms of data processing, is referred to as "nulling" mode. In this case, the probe is aligned with the flow direction so the angle of attack with respect to the central hole is set to zero. As a result, the central port is measuring the stagnation pressure of the flow, while the lateral ports are providing identical values, proportional to the static pressure. The aerodynamic center of the probe must be previously determined in the calibration setup ([5]). As a consequence, it requires of a highly-complex repositioning system leading to long acquiring times (the probe has to be aligned in each one of the measuring locations). Therefore, unsteady flows cannot be described using this method. The second operating mode, called "non-nulling", does not require any spatial alignment with respect to the flow incidence. In particular, the probe is placed at a constant flow angle (yaw) of the test section. Both magnitude and direction of the flow are determined according to a previous calibration that correlates the pressure values measured in the probe ports with the real flow conditions ([6]). When the probes are operating in "non-nulling" mode, it is possible to measure unsteady flows if the pressure transducers present an accurate frequency response. Since the flow inside turbomachinery passages is inherently unsteady, the paper will analyze in detail the 
performance of a CTHP operating in a non-nulling mode.

Practically, all the methods that can be found in the literature regarding the calibration procedure of multihole pressure probes ([7]) have a common feature: they are using normalized calibration coefficients. Such coefficients, as defined in [8], are obtained for a threehole probe as a function of the total pressure, $\mathrm{P}_{0}$, the static pressure, $\mathrm{P}_{\mathrm{S}}$, and all the pressures measured in the central hole, $\mathrm{P}_{\mathrm{C}}$, and in both left-hand side, $\mathrm{P}_{\mathrm{L}}$, and right-hand side holes, $\mathrm{P}_{\mathrm{R}}$, yielding:

$$
\begin{gathered}
\mathrm{C}_{\alpha}=\frac{\mathrm{P}_{\mathrm{L}}-\mathrm{P}_{\mathrm{R}}}{\mathrm{Q}} ; \quad \mathrm{C}_{\mathrm{Po}}=\frac{\mathrm{P}_{0}-\mathrm{P}_{\mathrm{C}}}{\mathrm{Q}} ; \quad \mathrm{C}_{\mathrm{PS}_{\mathrm{S}}}=\frac{\mathrm{P}_{0}-\mathrm{P}_{\mathrm{S}}}{\mathrm{Q}} \\
\mathrm{Q}=\mathrm{P}_{\mathrm{C}}-0.5\left(\mathrm{P}_{\mathrm{L}}+\mathrm{P}_{\mathrm{R}}\right)
\end{gathered}
$$

where $\mathrm{C}_{\alpha}$ represents the angular coefficient, $\mathrm{C}_{\mathrm{Po}}$ and $\mathrm{C}_{\mathrm{Ps}}$ are the total and static pressure coefficients and $\mathrm{Q}$ is the normalization factor that is introduced in all the coefficients. It is important to recall that is necessary to find a relationship between all the variables that are introduced in (1) in order to get accurate measurements of a particular flow field. Typically, this relationship can be found by means of a direct calibration of the probe ([9]).

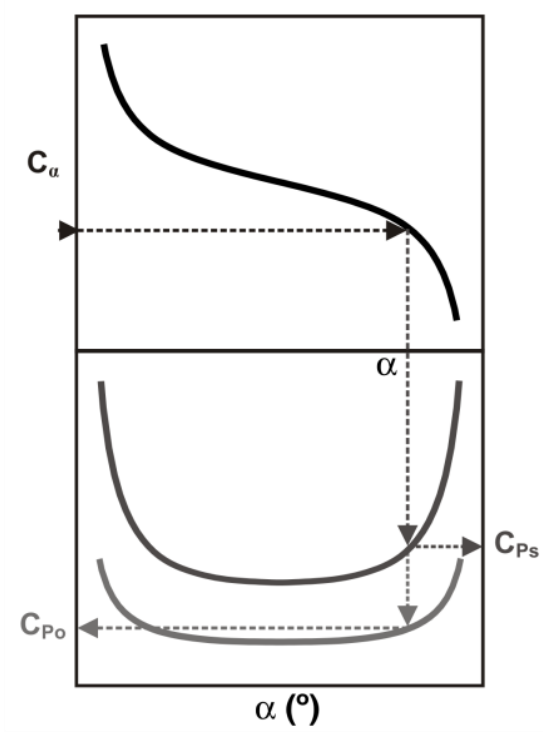

\section{Figure 3. Magnitude and direction of the flow obtained using a typical THP calibration.}

The calibration setup of a probe is based on a repositioning sequence of the probe inside a known flow field. This means that the relative angle position of the flow respect to the flow direction has to be progressively modified using a highly-precise angular stepping mechanism. For every angular position, the total and static pressures as well as all the pressure measurements in the holes are stored. Using these pressure values, all the calibration coefficients defined in (1) are directly obtained. Since the probe is symmetric, it is possible to fulfill the calibration just considering positive (or negative) incidence angles of the flow. However, in order to avoid slight differences derived from imperfections of the probe during the manufacturing process, it is preferred to conduct a complete calibration setup.

The calibration coefficients to be obtained following (1) and the procedure to determine both magnitude and direction of the flow after completing a measuring sequence with a CTHP is sketched in Fig. 3. Once the angular coefficient $\mathrm{C}_{\alpha}$ is calculated, the flow angle is known and the values of both pressure coefficients, $\mathrm{C}_{\mathrm{Po}}$ and $\mathrm{C}_{\mathrm{Ps}}$ are also determined. These coefficients allow us to calculate both static and total pressures of the flow. The difference between them, i.e. the dynamic pressure, is immediately related to the velocity magnitude of the flow.

Some authors have attempted to derive optimal mathematical fittings for the curves of the calibration coefficients. Different examples can be found in the literature, like references [10] and [11], where it is dealt the definition of third-order polynomial functions to represent local values of all the variables. The main goal is placed on the development of general relationships to avoid numerical tools that are necessary in the postprocessing. However, due to the improvement of the computational resources in recent years, it is always preferred to employ interpolation methods from direct measurements in the calibration sequence, rather than mathematical approaches with higher levels of inaccuracy in the computation of flow variables.

On the other hand, when this kind of multihole probes are calibrated with normalized coefficients (1), the key point is the establishment of the maximum operative ranges attainable for the probe. Basically, different criteria about the amplitude of their ranges can be defined if they are related to the angular range of the measurements, to the velocity range that is measurable and even to the frequency response of the probes.

Existing multihole pressure probes in classical references offer a quite diverse angular ranges for the measurements. As shown in references [4] and [12], three- and five-hole probes with angular ranges of $\pm 30^{\circ}$ and $\pm 25^{\circ}$ respectively present a limited overall performance. Large angular ranges have been obtained by some researchers (up to $\pm 70^{\circ}$ and, even, $\pm 80^{\circ}$ ), but through the design of probes with higher number of ports: seven holes in the case of references [8] and [13].

Differences in the angular range of the measurements -even for two probes with the same number of holes and angular distance between them- are due to the calibration procedure used when obtaining $\mathrm{C}_{\alpha}, \mathrm{C}_{\mathrm{Po}}$ and $\mathrm{C}_{\mathrm{Ps}}$. In order to reduce the number of samples in the calibration setup, it is mandatory that the angular coefficient $\mathrm{C}_{\alpha}$ varies monotonously with the flow angle $\alpha$. Otherwise, a unique value of the flow, $\alpha$, cannot be determined from the angular coefficient. Notice that the curve that defines the angular coefficient depends on the calibration procedure that is employed due to the normalization factor, Q. Anyway, there is always a maximum angle between the flow and the probe axis that implies that the flow is detached in two of the three holes of the probe ([14]). When this angle is exceeded, all the calibration data cannot be reduced to 
obtain the velocity field, so this is the upper limit of the angular range of the measurements.

Other typical problem that arises in the normalization with the coefficient $\mathrm{Q}$ is the introduction of singular points for all the pressure coefficients when $\mathrm{Q}$ turns to zero. In fact, the problem is not really associated to the apparence of singular points, which could be avoided easily by means of some kind of mathematical operator. The definitive problem lies in the non-monotonous behavior of the curve of the angular coefficient when operating close to the singularities.

Basically, the calibration method is based on the fact that when the pressure coefficients are normalized, they vary significantly with the flow incidence angle, but are practically independent of the flow velocity ([8]). The normalized calibration coefficients are obtained considering that the pressure distribution around a cylinder can be expressed as follows:

$$
\mathrm{P}=\mathrm{P}_{\mathrm{S}}+\mathrm{P}_{\mathrm{d}} \cdot \mathrm{C}_{\mathrm{p}}(\theta)
$$

where $\mathrm{P}_{\mathrm{d}}$ corresponds to the dynamic pressure, $\mathrm{C}_{\mathrm{p}}$ is the pressure coefficient and $\theta$ represents the angle between every point in the cylinder surface and the freestream direction. In the case of a three-hole probe, with a construction angle $\delta$, the pressure in every hole (central, left and right) is given by:

$$
\begin{gathered}
\mathrm{P}_{\mathrm{C}}=\mathrm{P}_{\mathrm{S}}+\mathrm{P}_{\mathrm{d}} \cdot \mathrm{C}_{\mathrm{p}}(\theta) \\
\mathrm{P}_{\mathrm{L}}=\mathrm{P}_{\mathrm{S}}+\mathrm{P}_{\mathrm{d}} \cdot \mathrm{C}_{\mathrm{p}}(\theta-\delta) \\
\mathrm{P}_{\mathrm{R}}=\mathrm{P}_{\mathrm{S}}+\mathrm{P}_{\mathrm{d}} \cdot \mathrm{C}_{\mathrm{p}}(\theta+\delta)
\end{gathered}
$$

If all the expressions in (3) are introduced in the definition of the normalized calibration coefficients (1), it is easily demonstrated that the coefficients are independent of both total and static pressures, being determined only by the pressure coefficient $\mathrm{C}_{\mathrm{p}}$.

The theoretical distribution of the pressure coefficients over a cylinder can be determined following the potential flow theory as a function of the angle $\theta$, according to:

$$
C_{p}=1-4 \sin ^{2} \theta
$$

which has been represented in Fig. 4 (inviscid line). Experimental distributions of the coefficient when both laminar and turbulent boundary layers (BL) are developed over the cylinder are also included in the figure. Noticeable differences can be found between the experimental results and the theoretical predictions. Thus, in the case of the laminar boundary layer, the flow separation overcomes at $\theta=82^{\circ}(15)$, inducing a wide wake with relatively low pressures, which results in a high value of the pressure coefficient. On the contrary, when the turbulent $\mathrm{BL}$ is set on, the separation arises for an angle of 120 degrees, the wake is narrower than before and higher pressures with a minor drag coefficient are found.
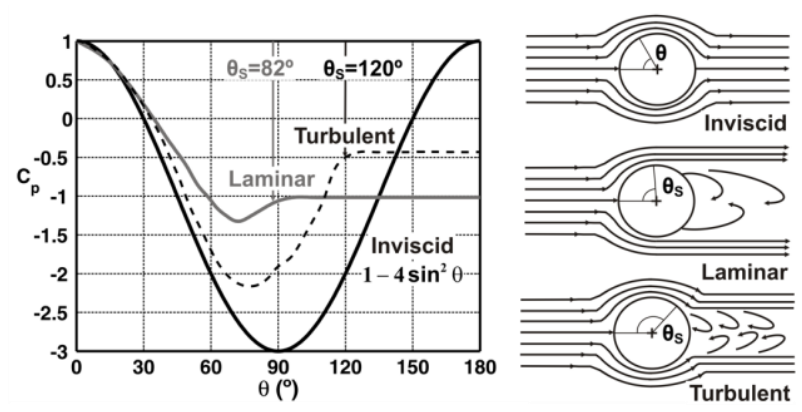

\section{Figure 4. Pressure coefficient around a cylinder:} inviscid, laminar and turbulent situations.

The potencial flow theory presents good agreement with the experimental results when $\theta$ is small, but important discrepancies appear when large angles are considered. In order to complete a rigorous analysis of all the calibration coefficients, a typical experimental distribution of $\mathrm{C}_{\mathrm{p}}$ has been introduced in the following section, instead of the potential law.

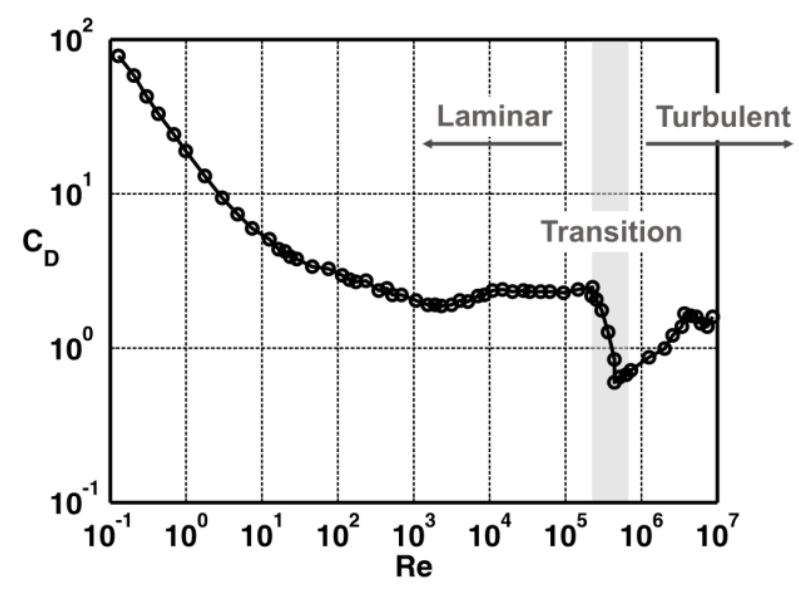

Figure 5. Drag coefficient as a function of the Reynolds number.

Figure 5 represents the variation of the drag coefficient for a cylinder as a function of the Reynolds number. This means that the distribution of the pressure coefficient is also a function of the flow velocity. However, it is possible to find large ranges where $C_{D}$ is practically constant. When $\mathrm{Re}$ is below $4 \cdot 10^{5}$, the $\mathrm{BL}$ presents laminar characteristics from the stagnation point to the locations where the flow is detached, with an important drag coefficient on the cylinder. When Re is between $4 \cdot 10^{5}$ and $7 \cdot 10^{5}$, there is a strong decay of the drag coefficient, usually known as "drag crisis", because of the instabilities of the flow arising from the critical number on. The transition to turbulent flow is characterized by a movement of the separation point towards the rear side of the cylinder, resulting in a narrower wake with lower drag. The variation of the $C_{D}$ when $\operatorname{Re}$ is between $10^{6}$ y $10^{7}$ is due to the transition to turbulent flow of those regions in the $\mathrm{BL}$ where the flow has not been detached earlier. Anyway, in the range between $10^{4}$ and $4 \cdot 10^{5}, \mathrm{C}_{\mathrm{D}}$ is practically constant, so it 
can be perfectly assumed that $C_{p}$ is independent of the velocity magnitude there.

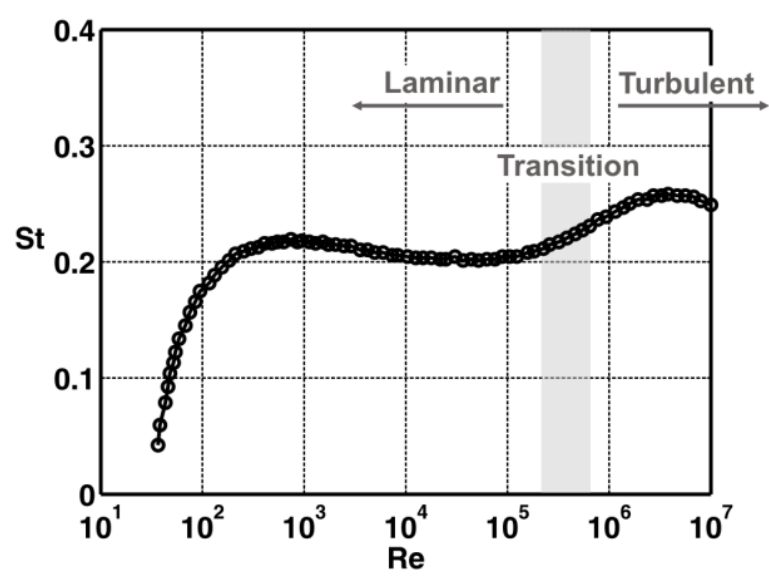

Figure 6. Strouhal number vs. Reynolds number.

The third limiting factor of the CTHP performance is referred to the frequency response of the probe. Though the flow around the probe may be steady, the wake behind the cylinder is inherently unstable, with an oscillating pattern that depends on the Reynolds number. The wake rolls up in vortices that are regularly shed from the cylinder. This kind of wakes is usually known as "Von Kàrman vortex street". The frequency associated to the vortex shedding is a function of the Strouhal number.

Figure 6 shows the variation of the Strouhal number with the Reynolds number (adapted from [15]). In the range between $10^{4}$ and $4 \cdot 10^{5}$, the Strouhal number is practically constant with a typical value of 0.2 . Beyond the shedding frequencies associated to a Strouhal number of 0.2 , the measurements of the probe are not capturing real flow physics. Instead, the sensors are measuring the Von-Kàrman vortex street or even oscillations of the probe itself.

\section{ANGULAR CALIBRATION}

This section contains a theoretical analysis of the calibration coefficients in the case of a CTHP with an angular distance of 45 degrees between the holes. The calibration coefficients that are obtained using a traditional normalizing factor $\mathrm{Q}$ are compared to the new set of coefficients derived from an improved normalization factor $\mathrm{Q}_{\mathrm{N}}$.

The distribution of the pressure coefficient $C_{p}$ in the central hole should be obtained rolling the probe 360 degrees about its own axis. However, since the interest is now placed in developing a theoretical framework of the calibration setup, the analysis is afforded using bibliographic data. Thus, we have employed for convenience a curve fitting tool based on splines through the experimental data collected in [16] for a Reynolds number of $2.3 \cdot 10^{4}$. The $\mathrm{C}_{\mathrm{p}}$ distributions in both left (L) and right (R) holes have been constructed shifting the original data for the central hole $\pm 45^{\circ}$ respectively. The final distributions in the three-holes, which are a function of the flow angle $\alpha$, are plotted in figure 7. Though adapted from experimental results, $C_{p}$ distributions imposed like this have to be considered as ideal, since these assumptions imply that there is no uncertainty associated either to the angular distance of the holes or to the probe misalignment.
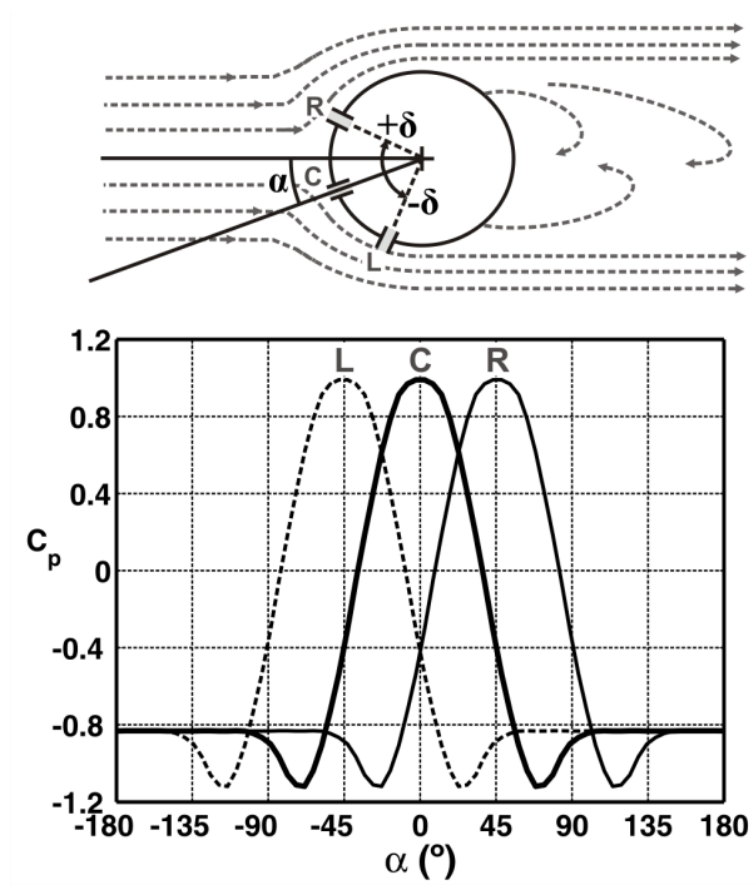

Figure 7. Pressure coefficients in the holes of a CTHP with an angular distance of $45^{\circ}$.

As expected, the maximum of the pressure coefficient in the central port is obtained for a flow angle of 0 degrees. Similarly, maximum values for both left and right ports are reached respectively at $\pm 45^{\circ}$. If the construction angle of the probe would be 60 degrees, then the maximum values for both lateral holes would be placed at $\pm 60^{\circ}$.

Following, with the pressure distributions of figure 7 , the calibration coefficients that are derived from the traditional calibration (normalization factor Q) are shown in figure 8.

The total and static pressure coefficients, $\mathrm{C}_{\mathrm{Po}}$ and $\mathrm{C}_{\mathrm{Ps}}$, are very similar. Both of them are symmetric respect to an incidence flow angle of 0 degrees. In the angular range of $\pm 30^{\circ}$ are positive, showing values between 0 and 4 . For the angular range of $\pm 10^{\circ}$ are nearly constant, rising as the flow angle increases towards $\pm 30^{\circ}$. On the contrary, the angular coefficient $\mathrm{C}_{\alpha}$ is anti-symmetric respect to a flow angle of 0 degrees. This way, it takes positive values for negative incidence angles and negative values for positive incidence angles, ranged from -6 to 6 . At a 0 degree flow angle, the angular coefficient is zero.

The significance of figure 8 is that the angular range of a CTHP calibrated with the traditional method is just limited to the interval between -30 and 30 degrees. Outside of this angular range, the angular coefficient is no longer monotonous with the flow angle, as illustrated in figure 9. Shown in the figure is the distribution of the 
angular coefficient calculated with the regular normalization factor $\mathrm{Q}$, for two probes with construction angles of 45 (black line) and 60 degrees (gray line). The $\mathrm{x}$-axis has been extended to include an angular range of $\pm 70^{\circ}$. Notice that outside the interval of $\left[-30^{\circ},+30^{\circ}\right]$, singular points are introducing sharp discontinuities in the coefficient. As a result, beyond the internal angular range, the calibration data cannot be reduced to obtain a unique flow angle, limiting the use of the probe.

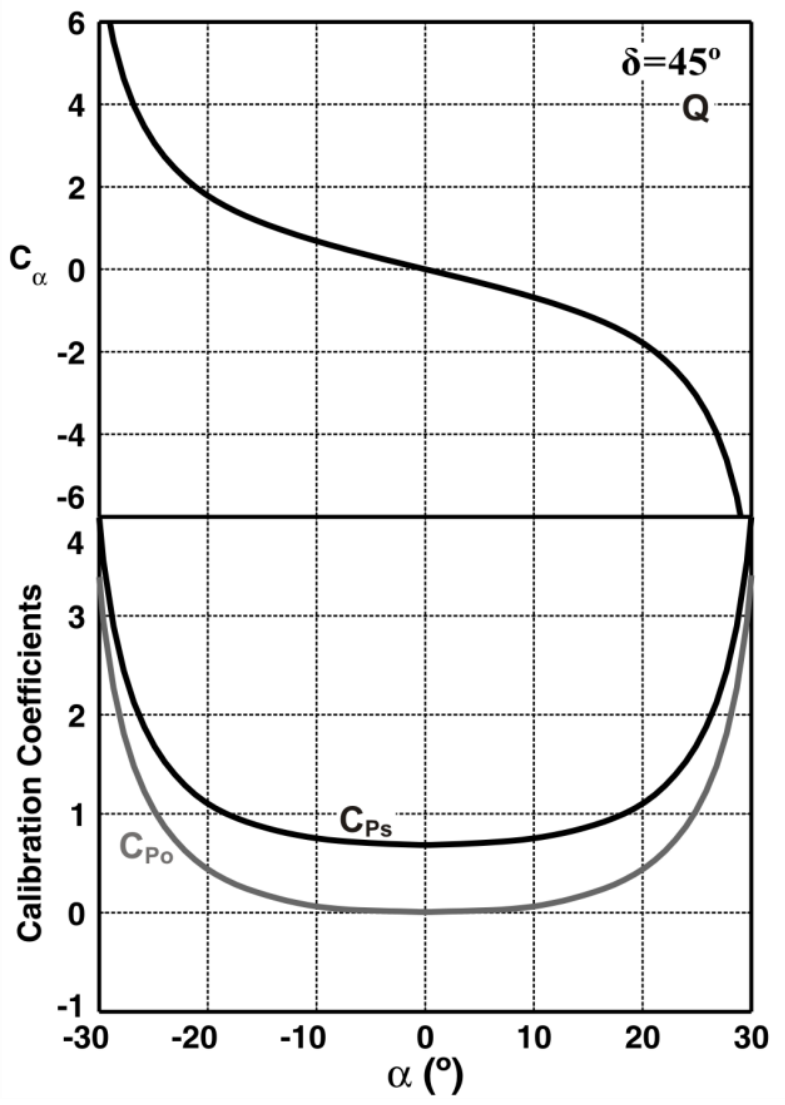

Figure 8. Calibration coefficients using the traditional normalization factor $Q, \delta=45^{\circ}$.

In addition, as observed in figure 9, both probes with different construction angles exhibit a similar attainable angular range. As a consequence of this result, an attempt was made to bring out a new method to calibrate a CTHP, with the final objective of increasing the angular range of the probes.

By definition, the angular coefficient is calculated as the pressure differential on the left and right holes, divided by the normalization factor -eq. (1)-. If the normalization factor would be value unity, the pressure differential between ports $\mathrm{L}$ and $\mathrm{R}$ would offer a monotonous angular coefficient along an angular range of, at least, twice the construction angle of the probe (see figure 7). With that pressure differential in the numerator of the angular coefficient, it was necessary to find a normalization factor as constant as possible when varying the flow angle. This new normalization factor is labelled as $\mathrm{Q}_{\mathrm{N}}$.

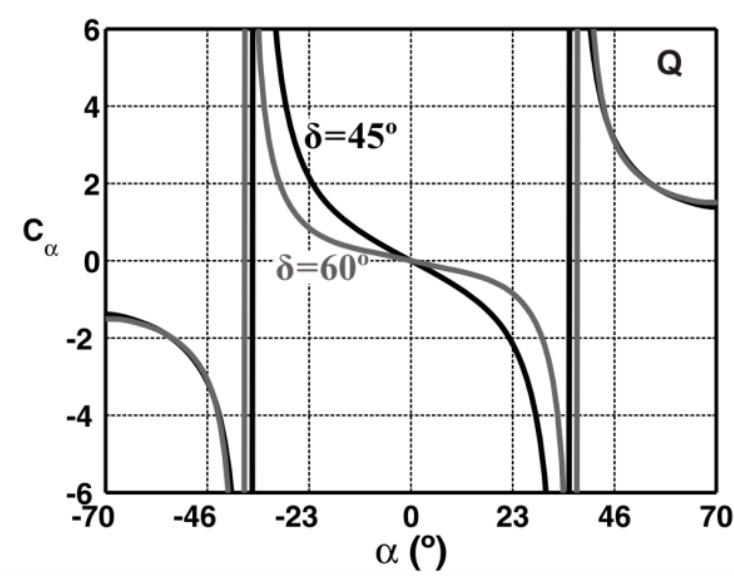

\section{Figure 9. Angular coefficient obtained using the normalization $Q$ for two CTHP with angular distances of $45^{\circ}$ and $60^{\circ}$.}

First of all, it is considered what happens in case of positive flow angles. In this case, the pressure distribution in the left hole is quite constant. Besides, up to 60 or 70 degrees, the pressure distribution in the central hole is quite similar to the inviscid behavior of potential flow (see figure 4), so it could be expressed as a function of $\cos ^{2} \alpha$. The pressure distribution in $R$ is obtained moving the central distribution an angular distance equal to the construction angle of the probe. As a consequence, it could be expressed as a function of $\sin ^{2} \alpha$. This trigonometric correlation for sine and cosine functions is valid in the angular range between 0 and 90 degrees, which includes the angular interval considered in this discussion. However, the objective is to find an approximate relationship between the pressure ports, rather than an exact mathematical expression. Then, since $\cos ^{2} \alpha+\sin ^{2} \alpha=1=$ cte, the new coefficient $Q_{N}$ could be defined as the sum of both pressures in $\mathrm{C}$ and $\mathrm{R}$. On the other hand, $\mathrm{Q}_{\mathrm{N}}$ must be independent of the static pressure. Considering that the pressure in $\mathrm{L}$ is nearly constant for positive flow angles, it is feasible to subtract twice the sum of the pressures in the central and right holes in order to eliminate the static pressure in the definition of $\mathrm{Q}_{\mathrm{N}}$. This yield:

$$
\mathrm{Q}_{\mathrm{V}}=\mathrm{P}_{\mathrm{C}}+\mathrm{P}_{\mathrm{R}}-2 \cdot \mathrm{P}_{\mathrm{L}} \text { for } \alpha>0^{\circ}
$$

This definition provides a normalization factor, independent of the static pressure, which can be expressed as the product of the dynamic pressure times a certain function of the flow angle. Thus, by means of $\mathrm{Q}_{\mathrm{N}}$, an angular coefficient independent of the dynamic pressure and only function of the flow angle is available.

Previous discussion is exclusively applicable for positive flow angles. In the case of negative incidences, it is necessary to reconsider this reasoning, so it can be deduced that the normalization factor should be now:

$$
Q_{\mathrm{V}}=\mathrm{P}_{\mathrm{C}}+\mathrm{P}_{\mathrm{L}}-2 \cdot \mathrm{P}_{\mathrm{R}} \text { for } \alpha>0^{\circ}
$$


In the calibration process, it is possible to define different angular coefficients in case of positive or negative flow angles, but when measuring this discrimination is unpractical. For a real measurement, you may have different angular coefficients only if you can differentiate the sign of the angular range using some of the pressure values sensed in the probe holes. Fortunately, in the case of the normalization factor $\mathrm{Q}_{\mathrm{N}}$ defined through (5) and (6), the flow angles are positive when $\mathrm{P}_{R}>\mathrm{P}_{L}$ and negative when $\mathrm{P}_{\mathrm{L}}>\mathrm{P}_{\mathrm{R}}$. Even when the flow angle is zero, both pressures in the left and right holes are the same, so $\mathrm{Q}_{\mathrm{N}}$ presents no discontinuities for $\alpha=0^{\circ}$. In summary, the calibration coefficient $Q_{N}$ is defined as:

$$
Q_{N}=\left\{\begin{array}{lll}
P_{C}+P_{R}-2 \cdot P_{L} & \text { for } & P_{R}>P_{L} \\
P_{C}+P_{L}-2 \cdot P_{R} & \text { for } & P_{L}>P_{R}
\end{array}\right.
$$

Figure 10 shows the angular coefficient that is calculated using $\mathrm{Q}_{\mathrm{N}}$ for two probes with an angular distance of 45 (black line) and 60 degrees (gray line) between the holes. The angular coefficient with this new method is monotonous, with a large angular range (up to $\pm 70^{\circ}$ ) and it is not presenting any singularity with the flow angle. Moderate values between -1.25 and 1.25 are exhibited by this new angular coefficient. In a similar fashion to previous traditional calibration, $\mathrm{C}_{\alpha}$ is negative in the case of positive flow angles and negative when the incidence is positive, being zero with no incidence angle.

Moreover, as shown in Fig. 10, both probes share a very similar attainable angular range $\left( \pm 70^{\circ}\right)$, independently of the angular separation $\delta$. The distribution of the angular coefficient is also very similar, though they present slight differences, especially in the slope at certain regions of the plot. Further insight in this concern will be made when analyzing the influence of the construction angle over the angular coefficient in following sections.

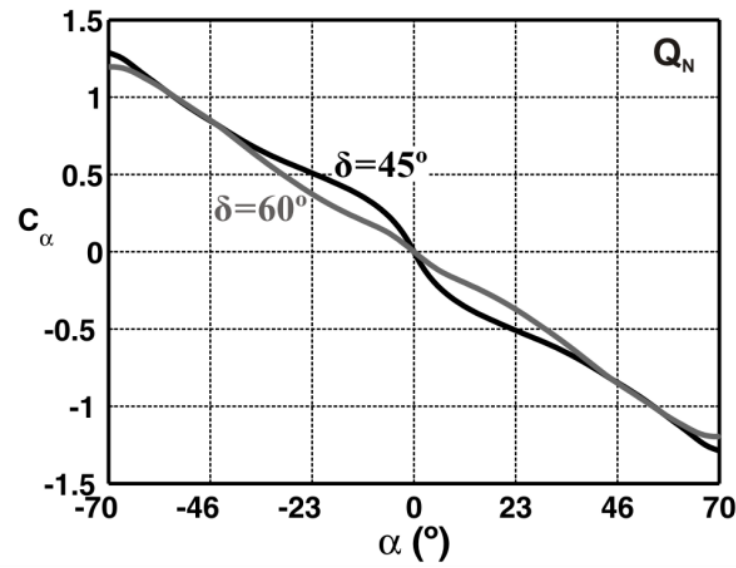

Figure 10. Angular coefficient obtained using the new normalization $Q_{N}$ for two CTHP with angular distances of $45^{\circ}$ and $60^{\circ}$.
The reason for an angular range of $\pm 70^{\circ}$ with no limitations in the construction angle of the CTHP is really a consequence of the variation of the normalization factor $\mathrm{Q}_{\mathrm{N}}$. When the magnitude of the pressure differential between $\mathrm{L}$ and $\mathrm{R}$ starts to decrease with the construction angle $\delta$, the normalization factor $\mathrm{Q}_{\mathrm{N}}$ is reduced as well, even in a faster rate. Therefore, the monotony of the angular coefficient with the flow angle is preserved, even after that the pressure differences L-R are no more a monotonous function of $\alpha$. If the normalization factor $\mathrm{Q}_{\mathrm{N}}$ would have been constant, the angular range would be severely limited by the angular separation between the holes.

The question arises as to why the angular range of the probe is precisely $\pm 70^{\circ}$, independently of the angular distance of the holes. Shown in figure 11 is a partial zoom of previous figure 7 pointing out the limits of the angular range. In the proximity to 70 degrees, the pressure in the left hole is practically constant. The pressure in the right hole begins to fall from higher levels while the pressure in $\mathrm{C}$ has reached its minimum and now begins to increase. This implies that from the point with the minimum pressure value in $\mathrm{C}$ on, it is not possible to discriminate the pressure values, leading to the appearance of unacceptable double solutions for the flow angle. This assertion is a purely mathematical conclusion, based on the fact that the three-equation linear system given in (3) has no unique solution when the flow angle is outside the angular range of $\pm 70^{\circ}$. Also, when the pressure values in $\mathrm{C}$ and $\mathrm{L}$ are approximately equal to each other, the uncertainty of the measurement is dramatically increased and the probe resolution fails. Additional insight on this topic will be presented in the next section.

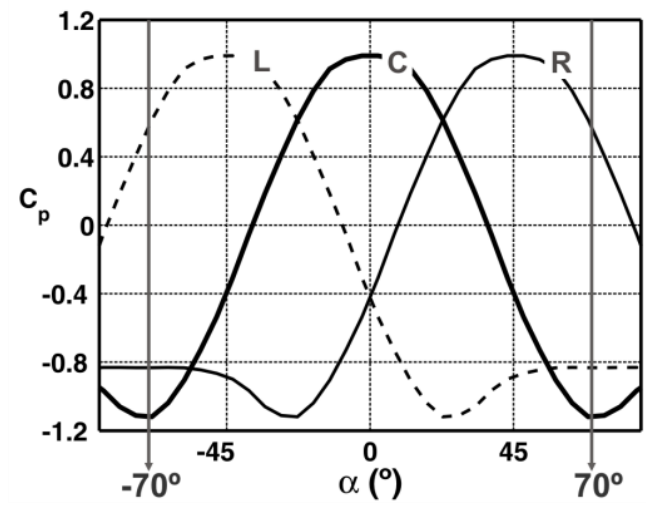

\section{Figure 11. Limits of the angular range for a CTHP calibrated with the normalization factor $Q_{N}$.}

Summarizing, the great advantage of this new method (based in the normalization factor $\mathrm{Q}_{\mathrm{N}}$ ) to calibrate a CTHP is that the angular range of the probe is improved in 80 degrees. Therefore, it is possible to dispose of a pressure probe that allows measuring over a large range of flow onset angles, in particular up to 140 degrees. 


\section{UNCERTAINTY}

Having defined this new calibration method, it is necessary to analyze the uncertainty levels associated to the normalization factor $\mathrm{Q}_{\mathrm{N}}$. Present investigations confirm that not only the angular range is increased, but also the uncertainty levels are roughly different respect to typical acceptable tolerances.

In many ways, the factor $Q_{N}$ can be considered as two times the traditional coefficient $Q$, since it is calculated using twice the pressure values of the holes. Then, the uncertainty of the measurements associated to the coefficient $\mathrm{Q}_{\mathrm{N}}$ may be estimated somehow as twice the uncertainty of the measurements calibrated using $Q$. Nevertheless, the uncertainty in the angular coefficient is small because of the reduced transfer of uncertainty in the mathematical process. In fact, though the uncertainty in the flow angle is higher (shown later) for small flow angles, the uncertainty in the velocity magnitude and the static pressure are lower, even inside the typical angular range of traditional calibration (between $\pm 10^{\circ}$ and $\pm 30^{\circ}$ ).

The methodology proposed in [17] has been followed to evaluate the uncertainty of the flow variables measured with a CTHP. Hence, according to previous distributions of the angular coefficient, the flow angle can be expressed in terms of a unique explicit analytic function of $\mathrm{C}_{\alpha}$. Mathematically speaking:

$$
\mathrm{I}_{\alpha}^{2}=\left(\frac{\partial \alpha}{\partial \mathrm{C}_{\alpha}}\right)^{2} \mathrm{I}_{\mathrm{C}_{\alpha}}^{2}
$$

Using the definition of the angular coefficient in (1), the uncertainty of $\mathrm{C}_{\alpha}$ can be also obtained following an analogous algebra:

$$
\begin{gathered}
\mathrm{I}_{\mathrm{C}_{\alpha}}^{2}=\left[\left(\frac{\partial \mathrm{C}_{\alpha}}{\partial \mathrm{P}_{\mathrm{L}}}\right)^{2}+\left(\frac{\partial \mathrm{C}_{\alpha}}{\partial \mathrm{P}_{\mathrm{R}}}\right)^{2}+\left(\frac{\partial \mathrm{C}_{\alpha}}{\partial \mathrm{Q}}\right)^{2}\right] \cdot \mathrm{I}_{\mathrm{P}}^{2}= \\
=\frac{1}{\mathrm{Q}^{2}}\left[2 \mathrm{I}_{\mathrm{P}}^{2}+\mathrm{C}_{\alpha}^{2} \mathrm{I}_{\mathrm{Q}}^{2}\right]
\end{gathered}
$$

where $\mathrm{Q}$ is the traditional normalization factor. To that end, it has been supposed that the uncertainty in the pressure is the same for all the holes, being equal to the uncertainty in a pressure measurement, $I_{P}$, which is basically determined by the uncertainty of the pressure transducers.

The uncertainty of the traditional normalization factor $\mathrm{Q}$ is given by:

$$
\begin{gathered}
\mathrm{I}_{\mathrm{Q}}^{2}=\left[\left(\frac{\partial \mathrm{Q}}{\partial \mathrm{P}_{\mathrm{C}}}\right)^{2}+\left(\frac{\partial \mathrm{Q}}{\partial \mathrm{P}_{\mathrm{L}}}\right)^{2}+\left(\frac{\partial \mathrm{Q}}{\partial \mathrm{P}_{\mathrm{R}}}\right)^{2}\right] \cdot \mathrm{I}_{\mathrm{P}}^{2}= \\
=\frac{3}{2} \cdot \mathrm{I}_{\mathrm{P}}^{2}
\end{gathered}
$$

Substituting equation (10) into (9), the uncertainty of the angular coefficient is expressed as a function of the uncertainty in the pressure measurement:

$$
\mathrm{I}_{\mathrm{C}_{\alpha}}^{2}=\left[2+\frac{3}{2} \mathrm{C}_{\alpha}^{2}\right] \frac{\mathrm{I}_{\mathrm{P}}^{2}}{\mathrm{Q}^{2}}
$$

Finally, including equation (11) in (8), the uncertainty of the flow angle is:

$$
I_{\alpha}=\left(\frac{\partial \alpha}{\partial C_{\alpha}}\right) \cdot \sqrt{2+\frac{3}{2} C_{\alpha}^{2}} \cdot \frac{I_{P}}{Q}
$$

where the derivative of the angle with respect to the angular coefficient must be evaluated numerically. Analogous considerations lead to define the relative uncertainty for the velocity magnitude as well as the static pressure uncertainty, according to:

$$
\begin{gathered}
\frac{I_{v}}{v}=\sqrt{\frac{3}{2} \cdot \frac{C_{P s}}{2 P_{d}} \cdot I_{P}} \\
I_{P s}=\sqrt{1+\frac{3}{2}\left[C_{P_{o}}^{2}+C_{P s}^{2}\right]} \cdot I_{P}
\end{gathered}
$$

Applying identical deductions in the case of the new calibration method, the uncertainty of the normalization factor $\mathrm{Q}_{\mathrm{N}}$ is:

$$
\begin{gathered}
\mathrm{I}_{\mathrm{Q}_{\mathrm{N}}}^{2}=\left[\left(\frac{\partial \mathrm{Q}_{\mathrm{N}}}{\partial \mathrm{P}_{\mathrm{C}}}\right)^{2}+\left(\frac{\partial \mathrm{Q}_{\mathrm{N}}}{\partial \mathrm{P}_{\mathrm{L}}}\right)^{2}+\left(\frac{\partial \mathrm{Q}_{\mathrm{N}}}{\partial \mathrm{P}_{\mathrm{R}}}\right)^{2}\right] \cdot \mathrm{I}_{\mathrm{P}}^{2}= \\
=6 \cdot \mathrm{I}_{\mathrm{P}}^{2}
\end{gathered}
$$

From (15), all the uncertainties associated to the flow angle, the velocity magnitude and the static pressure for the new calibration are given by:

$$
\begin{gathered}
\mathrm{I}_{\alpha}=\left(\frac{\partial \alpha}{\partial \mathrm{C}_{\alpha}}\right) \cdot \sqrt{2+6 \mathrm{C}_{\alpha}^{2}} \cdot \frac{\mathrm{I}_{\mathrm{P}}}{\mathrm{Q}_{\mathrm{N}}} \\
\frac{\mathrm{I}_{\mathrm{v}}}{\mathrm{v}}=\sqrt{\frac{3}{2} \cdot \frac{\mathrm{C}_{\mathrm{Ps}}}{\mathrm{P}_{\mathrm{d}}} \cdot \mathrm{I}_{\mathrm{P}}} \\
\mathrm{I}_{\mathrm{Ps}}=\sqrt{1+6\left[\mathrm{C}_{\mathrm{Po}}^{2}+\mathrm{C}_{\mathrm{Ps}}^{2}\right]} \cdot \mathrm{I}_{\mathrm{P}}
\end{gathered}
$$



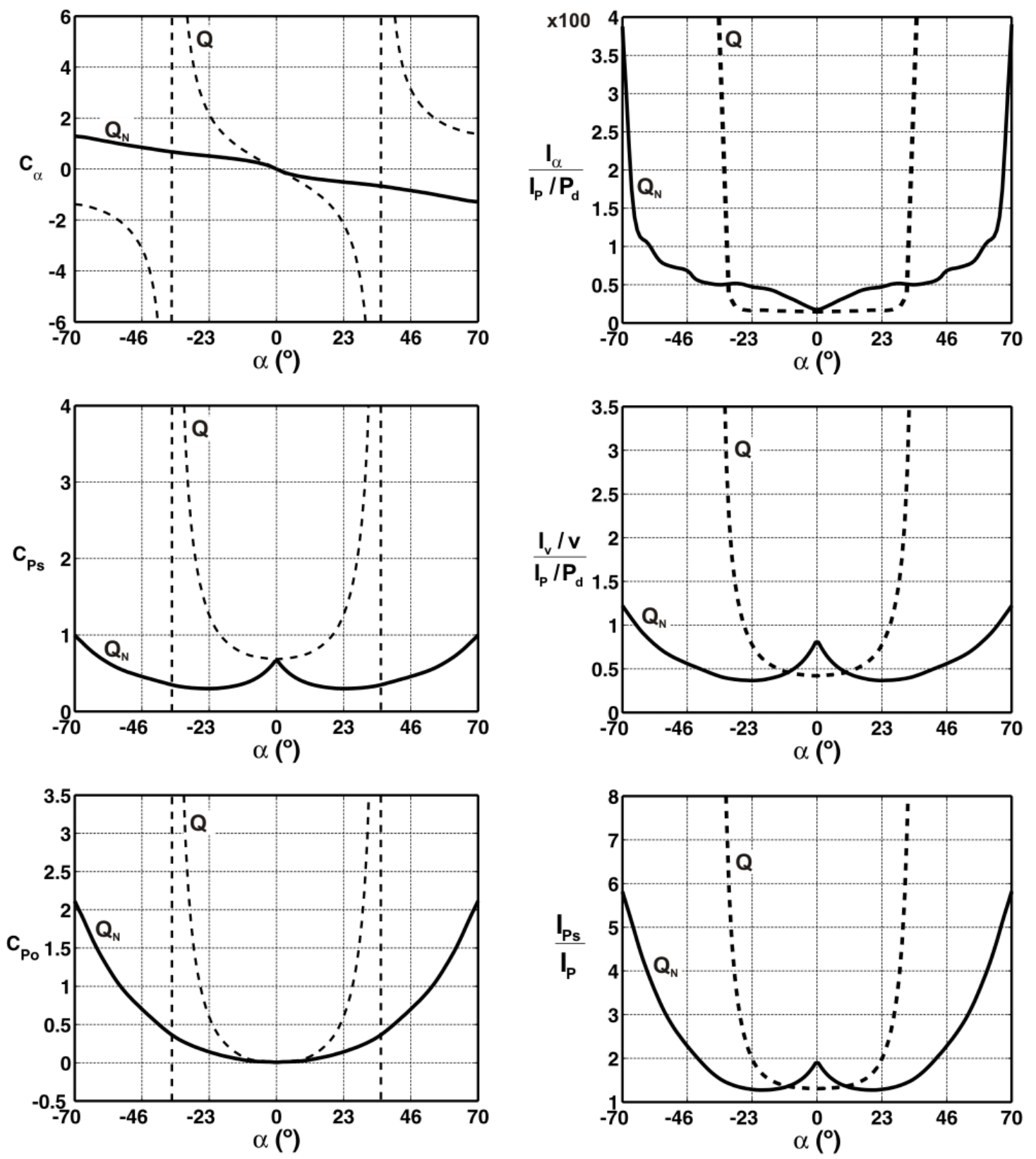

Figure 12. Calibration coefficients and uncertainty levels in a CTHP with $\delta=45^{\circ}$ obtained through traditional $(Q)$ and improved $\left(Q_{\mathrm{N}}\right)$ calibrations.

The right column of figure 12 shows the distribution of all the uncertainties formulated before as a function of the flow angle. Both results for traditional (dashed lines) and new (solid lines) calibrations are included in the array of plots. In addition, the left column of the figure reproduces the calibration coefficients that are derived from both normalization factors $\mathrm{Q}$ and $\mathrm{Q}_{\mathrm{N}}$. All the uncertainties have been made non-dimensional. The uncertainty of the flow angle is expressed as a percentage of the pressure uncertainty, $I_{P}$, relative to the dynamic pressure $P_{d}$. This means that, for instance, if $\alpha=30^{\circ}$ the uncertainty in the flow angle is about 0.5 degrees for every $1 \%$ of $\mathrm{I}_{\mathrm{P}} / \mathrm{P}_{\mathrm{d}}$. The uncertainty for the static pressure is referenced to the uncertainty of the pressure measurement. Finally, the relative uncertainty for the velocity has been made non-dimensional with the relative uncertainty of the dynamic pressure, instead of using the static pressure. This implies that the static pressure of the flow cannot be excessively high respect to the dynamic pressure.

From figure 12, it is observed a common feature for both calibrations: the uncertainty of the flow angle tends to infinite when the normalization coefficient tends to zero (at $\pm 30^{\circ}$ for $\mathrm{Q}$ and $\pm 70^{\circ}$ for $\mathrm{Q}_{\mathrm{N}}$ ). This is because the angle uncertainty is calculated dividing by the normalization factor -equations (12) and (16)-. Alternatively, though the relative uncertainty of the velocity and the uncertainty of the static pressure are not obtained dividing by the normalization factor equations (13), (14), (17) and (18)-, both variables tend to infinite at $\pm 30^{\circ}$ when using the traditional calibration. This is a consequence of the behavior of 
both static and total pressure coefficients, that are also infinite at $\pm 30^{\circ}$ (left column of the figure). On the contrary, the relative uncertainty of the velocity and the uncertainty of the static pressure in case of the new calibration are increased towards $\pm 70^{\circ}$, but limited to finite values.

For a flow angle of zero degrees, the uncertainty in the angle measurement is the same for both calibrations. Inside the angular range of $\pm 30^{\circ}$, the uncertainty $I_{\alpha}$ is slightly higher when using the new calibration than the traditional one. Even so, at $\pm 60^{\circ}$ it is considerable small with just $1^{\circ}$ for every $1 \%$ of $\mathrm{I}_{\mathrm{P}} / \mathrm{P}_{\mathrm{d}}$.

The relative uncertainty in the velocity magnitude and the uncertainty in the static pressure are a bit higher when calibrating with $\mathrm{Q}_{\mathrm{N}}$ for $\alpha=0^{\circ}$. On the other hand, in the angular intervals $\left[-30^{\circ},-10^{\circ}\right]$ and $\left[10^{\circ}, 30^{\circ}\right]$, the results show a better performance of the new calibration method. Complementarily, the relative uncertainty of velocity takes values ranged between 0.5 and 1.2 times $\mathrm{I}_{\mathrm{P}} / \mathrm{P}_{\mathrm{d}}$ for the whole angular range, which is an exceptional good result. The uncertainty of the static pressure is strongly increased from 1.2 to 2 times $I_{P}$ at $\pm 40^{\circ}$, reaching up to 6 times $I_{P}$ when being nearby $\pm 70^{\circ}$.

Anyway, overall differences between the uncertainties evaluated for both calibration methods are not significant in the angular range of $\pm 30^{\circ}$. Therefore, the new calibration is not only providing a CTHP that is capable of measuring angular variations of the flow up to 140 degrees, but it also presents reasonable low uncertainties for the whole angular range of the probe.

\section{INFLUENCE OF THE ANGULAR DISTANCE BETWEEN THE HOLES}

Having observed that with the new normalization factor $\mathrm{Q}_{\mathrm{N}}$, the attainable angular range of a CTHP is practically the same for a construction angle of 45 that for an angle of 60 degrees, it led to investigate if it is possible to find an optimal construction angle $\delta$ in order to minimize the uncertainty in the measurements.

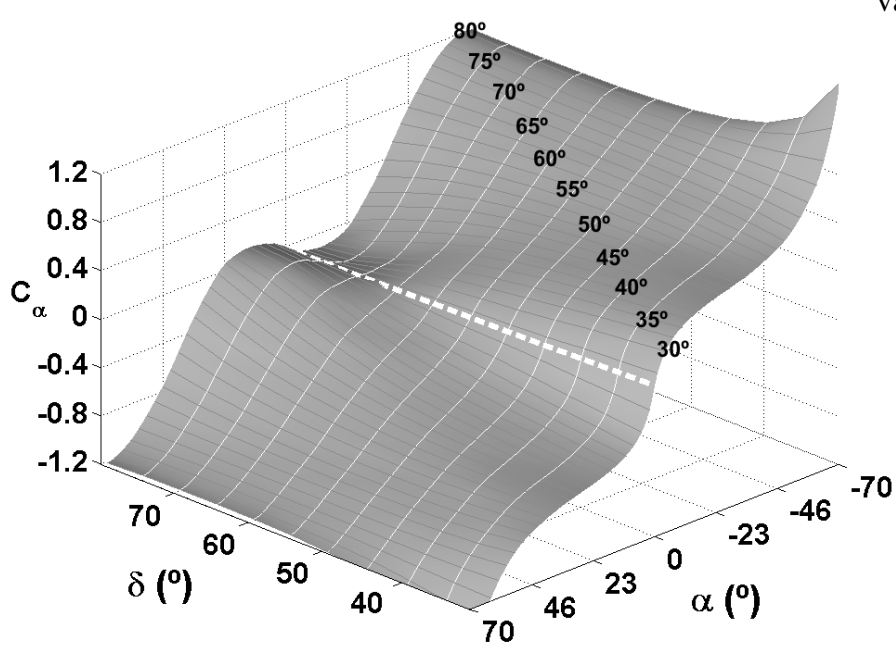

Figure 13. Angular coefficients obtained from the new calibration $\left(Q_{N}\right)$ for a CTHP as a function of the angular distance $\delta$ between the holes.
Figure 13 shows the angular coefficient normalized with $\mathrm{Q}_{\mathrm{N}}$ as a double function of the flow angle and the angular distance between the holes. In this case, beyond a separation distance of 70 degrees, the angular coefficient of the probe losses its monotonous behavior around a flow angle of 0 degrees, so the calibration cannot provide a unique value and fails. This characteristic is also reproduced for angular distances of the holes below 25 degrees (not shown in the figure). Hence, the theoretical limits for these construction angles of the probe are between 25 and 70 degrees.

Probes designed with the lowest separation angles are presenting angular coefficients with a higher slope when the flow angle is approaching to zero degrees. However, within the range of 50 to 60 degrees for the angular separation of the holes, the slope of the angular coefficients is more uniform for the whole range of flow angles. A priori, a uniform slope of the angular coefficient implies a lower uncertainty in the measurements, since the uncertainty of the angle measurement is obtained from that slope -equation (16)-. Therefore, it is quite suspectable that, in terms of uncertainty, the optimal separation angles between the holes of the probe must be chosen between 50 and 60 degrees. Respect to the horizontal range, though small variations in the maximum angular range of the measurements are observed in figure 13 (the different tails of all the distributions), they are sufficiently small to be omitted.

Through the complete set of formulae developed for the new calibration in the previous section, the uncertainty in the measurements for the flow angle, the velocity magnitude and the static pressure have been represented as a function of both flow angle, $\alpha$, and angular distance, $\delta$, in the left column of figure 14 .

Since there are excessive variations in the uncertainties as a function of both angles, the particular case for a flow angle of zero degrees has been extracted from the three-dimensional map and plotted in the right column of the figure. These plots are also including maximum values of uncertainty and mean values averaged for every angular distance. To enhance

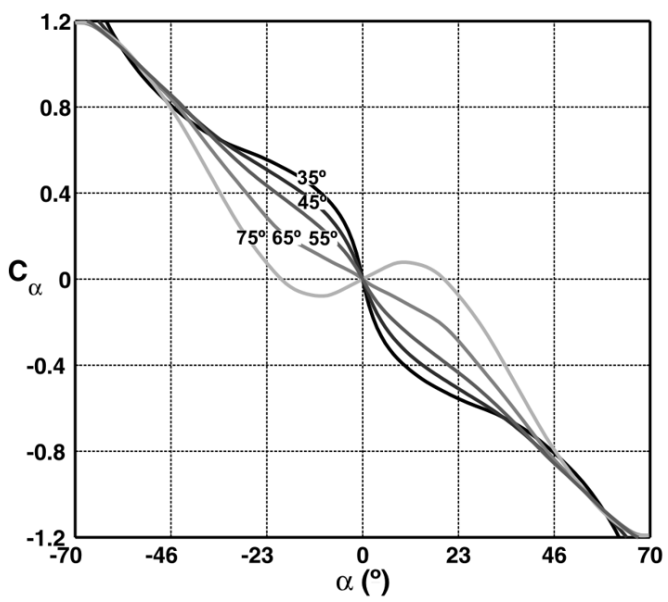


the visualization of the results, the three-dimensional maps are only showing the values within an angular range of $\pm 60^{\circ}$, since the uncertainty levels are dramatically increased when the flow angle is outside that range.

The uncertainty of the angle measurement is quite constant for angular distances of the holes between 30 and 65 degrees. For a flow incidence of zero degrees (dashed lines) it takes values around 0.25 degrees for every $1 \%$ of $\mathrm{I}_{\mathrm{P}} / \mathrm{P}_{\mathrm{d}}$. The mean uncertainty (solid black lines) is limited to 0.5 degrees for every $1 \%$ of $\mathrm{I}_{\mathrm{P}} / \mathrm{P}_{\mathrm{d}}$, while the maximum uncertainty (gray lines) is maintained about 1 degree for every $\mathrm{I}_{\mathrm{P}} / \mathrm{P}_{\mathrm{d}}$. Beyond an angular distance of 65 degrees for the holes, the uncertainties tend to be infinite.
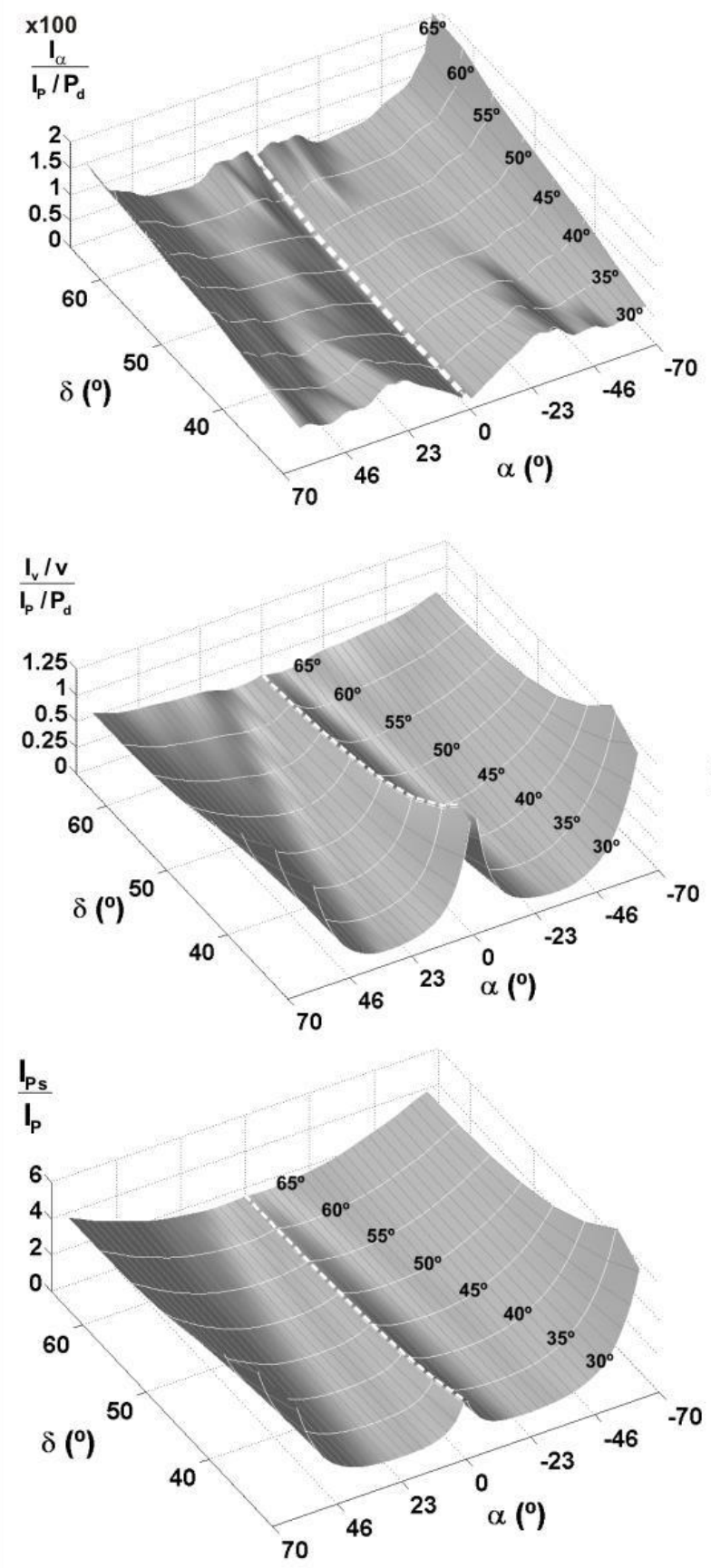

The mean value of the relative uncertainty for velocity is basically constant, averaging 0.6 times $I_{p} / P_{d}$. In a similar fashion, the mean value of the uncertainty for the static pressure is placed around 2 times $I_{P}$ for the whole range of construction angles. In addition, in case of both velocity and static pressure uncertainties, the maximum values and the distribution when $\alpha=0^{\circ}$ are increased if the angular distance between the holes is reduced. When $\alpha=0^{\circ}$, the relative uncertainty of the velocity is placed between 0.6 and 1.5 times $\mathrm{I}_{\mathrm{P}} / \mathrm{P}_{\mathrm{d}}$, while the uncertainty of the pressure varies from 1.5 to 3.2 times $\mathrm{I}_{\mathrm{P}}$. Finally, the results show that maximum values of the velocity uncertainty overlap with the values of zero-degree flow angle when the angular
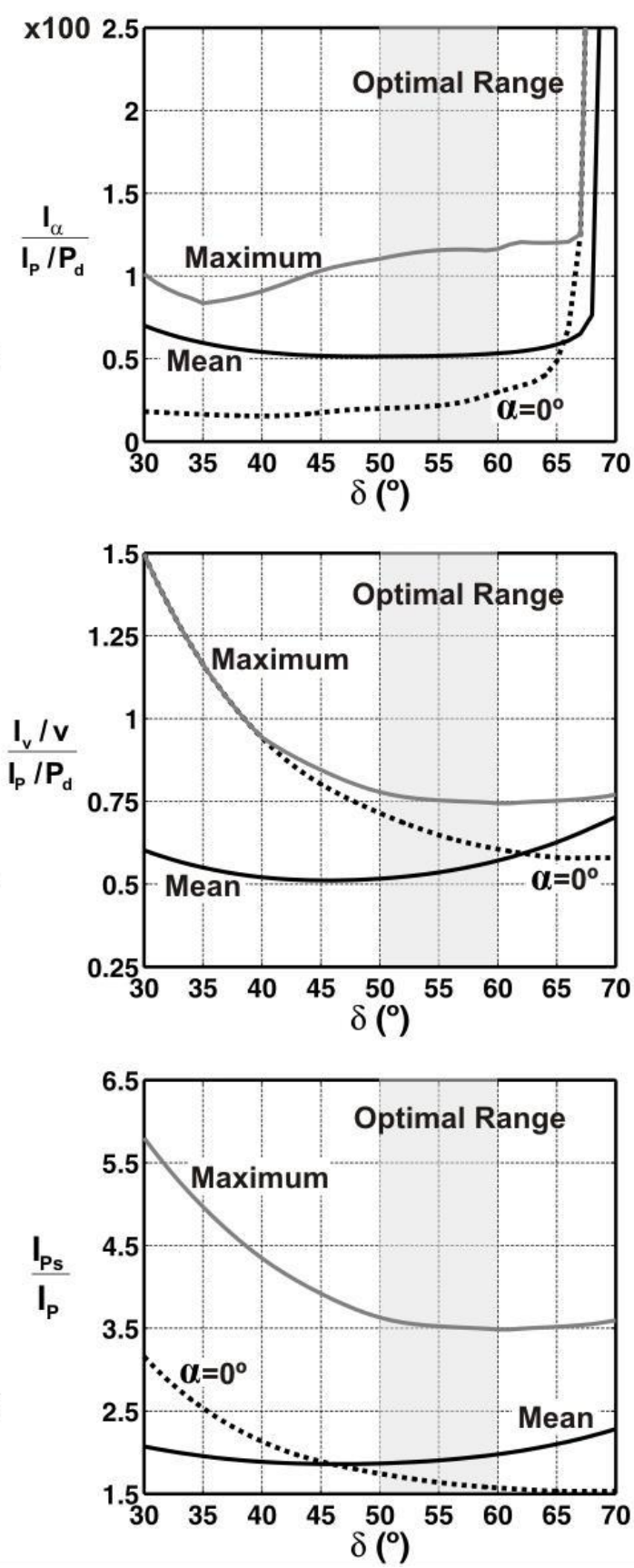

Figure 14. Uncertainty of a CTHP calculated from the new calibation $\left(Q_{\mathrm{N}}\right)$ as a function of both flow angle, $\alpha$ and the angular distance between the holes, $\delta$. 
distance of the holes is below 40 degrees. The values range between 0.75 and 1.5 times $\mathrm{I}_{\mathrm{P}} / \mathrm{P}_{\mathrm{d}}$. In the case of the static pressure, maximum values of uncertainty are placed between 3.5 and 5.7 times IP. In any case, all the uncertainty ranges of the probe are perfectly acceptable for typical applications (e.g., turbomachinery, boundary layers, free shear flows).

As a conclusion, figure 14 points out that, in terms of accuracy of the probe uncertainty, the more desirable design of the probes is achieved when the angular distance between the holes is chosen to be from 50 to 60 degrees ("optimal range" in the figure). However, other designs with angular separation ranged from 45 to even 65 degrees are also perfectly valid, since the angular range is practically conserved and the uncertainty of the measurements is still well-restricted.

\section{EXPERIMENTAL APPLICATION}

As a practical application, a specific-designed CTHP to measure the flow downstream of the rotor of an axial turbomachine, has been developed and calibrated according to the new method presented in this paper. The axial turbomachine is a single-staged, low-speed axial fan with a typical stator-rotor configuration.

The main objective of this experiment is to measure a highly-complex, incompressible flow which is roughly uniform in the radial plane (at midspan) but presents large non-uniformities in the circumferential direction. Therefore, in the central positions of the blade passages, the flow can be assumed to be twodimensional, with a typical wake-jet structure that is periodically modifying the flow angle at the exit. Consequently, the flow patterns can be perfectly described by means of a typical two-dimensional pressure probe like a CTHP.

The rotor of the turbomachine is rotating in front of the probe location, so the probe is alternatively facing fluid flow coming from the free-stream region of the blade passages and fluid flow coming from the rotor wakes. Since the wake fluid presents and important velocity deficit associated to the blade blockage, there are important variations in both magnitude and direction of the flow in every blade passing period.

The probe frequency response that is necessary to acquire the typical length and time scales of this phenomenon, must be at least ten times the blade passing frequency (BPF). In addition, in the analysis of the CTHP uncertainty, it was assumed that the static pressure cannot be excessively large compared to the dynamic pressure. Effectively, downstream of the rotor, the static pressure is hardly modified in the tangential direction, but slightly changing in the radial direction. This means that a CTHP calibrated using the new normalization factor is perfectly accurate to describe a fluid flow with these characteristics.

Figure 15 shows a sketch of the pressure probe designed to measure the flow patterns of the axial flow fan. In particular, the diameter of the probe is $8 \mathrm{~mm}$ with a total length of $75 \mathrm{~cm}$. With these dimensions, the Reynolds number based on the probe diameter and the free-stream velocity is around $2 \cdot 10^{4}$. The probe was designed for an angular separation of 60 degrees between the holes, which are placed at $15 \mathrm{~mm}$ from the semi-spherical tip. The hole-labelling convection is $\mathrm{C}$, $\mathrm{L}$ and $\mathrm{R}$ for central, left and right holes as usual. Previous experiments were conducted using a similar probe, but with an angular distance of just 45 degrees. Respect to the probe holes, they are containing miniature pressure sensors of $2.3 \mathrm{~mm}$ diameter and 16 $\mathrm{mm}$ length. The cylindrical geometry of the probe allows placing the transducers in an equally spaced layout of 120 degrees around the probe axis. This arrangement implies a minimum distance between the transducers, so it is feasible to construct a probe with the diameter as small as possible. The distance from the holes face to the transducers is $4 \mathrm{~mm}$ for both left and right ports, while it is increased to $8 \mathrm{~mm}$ in the central port (see the top view in the sketch). Since the holes are so close one to each other, the frequency response of the probe is just depending on the transducers sensibility.
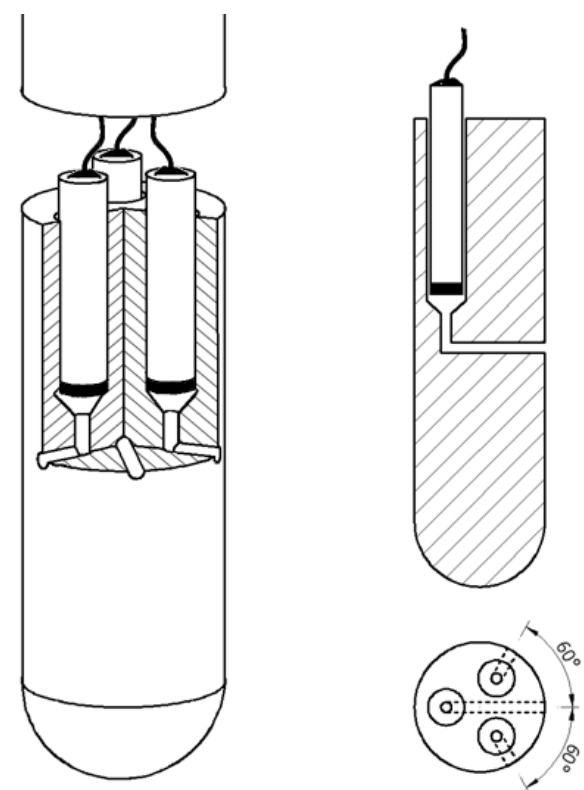

Figure 15. Sketch of the specific-designed CTHP to measure the flow downstream of the rotor.

Figure 16 shows a typical experimental calibration, obtained using the new normalization factor $\mathrm{Q}_{\mathrm{N}}$, for the CTHP. The calibration was carried out in a stepping rotational mechanism using angular intervals of 10 degrees. Though this sequence is not very precise, it is useful to appreciate the calibration data. For instance, with these coarse distributions, it is possible to expose that the probe is slightly misaligned within the holder, approximately 5 degrees. Notice that using a calibration method based on mathematical curve fitting, all the coefficients would be approached to theoretical distributions. As a consequence, this slight misalignment would derive in a classical bias error. By means of the calibration methodology presented in this paper, this error is vanished because it is really integrated with the information of the calibration. 


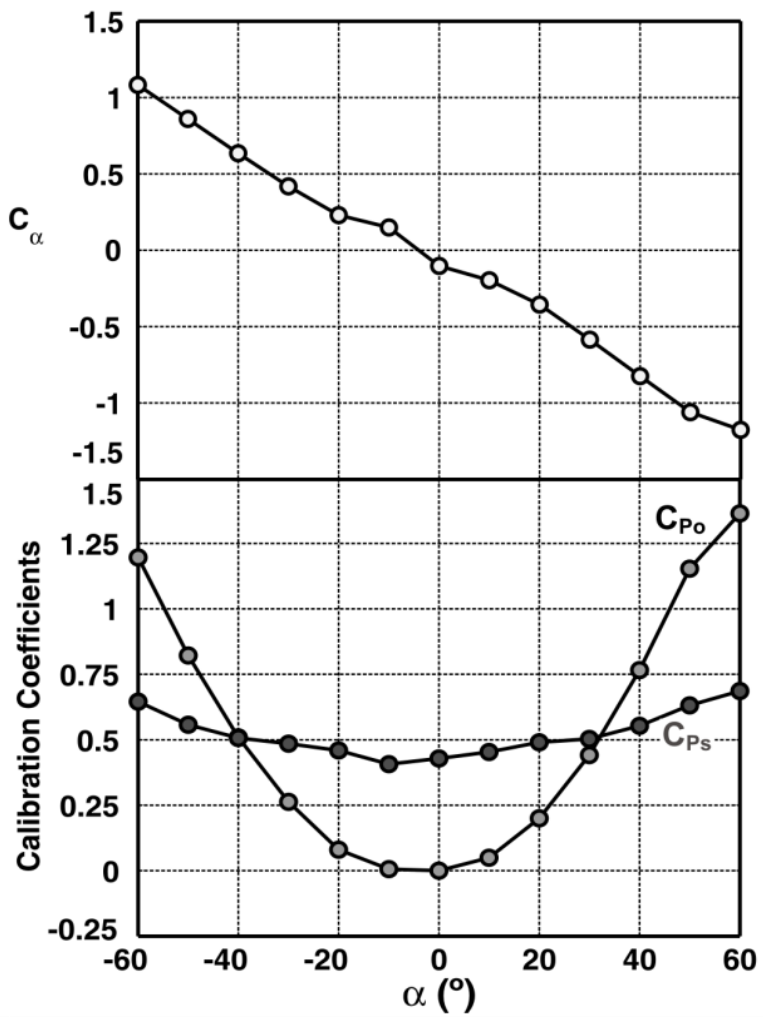

Figure 16. Experimental calibration according to normalized coefficients, $Q_{\mathrm{N}}$, for a CTHP with $\delta=60^{\circ}$.

Figure 17 shows the results of an instantaneous measurement downstream of the fan rotor. The pressure signals acquired in the holes have been already translated to values of velocity magnitude and flow angle.

The velocity magnitude has been made nondimensional with the blade tip velocity. The flow angle is referred to the probe axis. The horizontal axis represents time, non-dimensionalized by rotor blade passing period, $\mathrm{T}_{\mathrm{r}}$. At a first glance, though the signals are extremely turbulent, it is possible to identify the rotor wakes in the angle signal, with large variations ranging from $-20^{\circ}$ to $45^{\circ}$. Moreover, some other traces (not shown here) have presented even higher variations of the flow angle between the wake fluid and the freestream flow. Obviously, this justifies the election of CTHP calibrated in order to obtain large angular resolution.

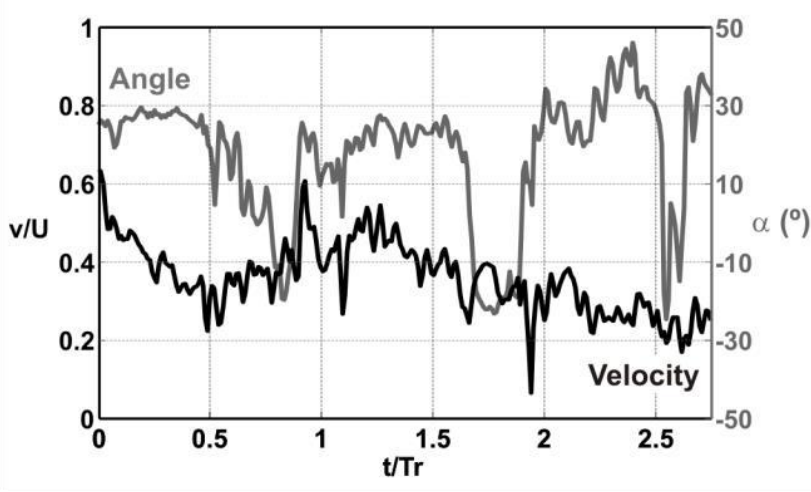

Figure 17. Instantaneous measurement of the velocity magnitude and the flow angle with a CTHP.
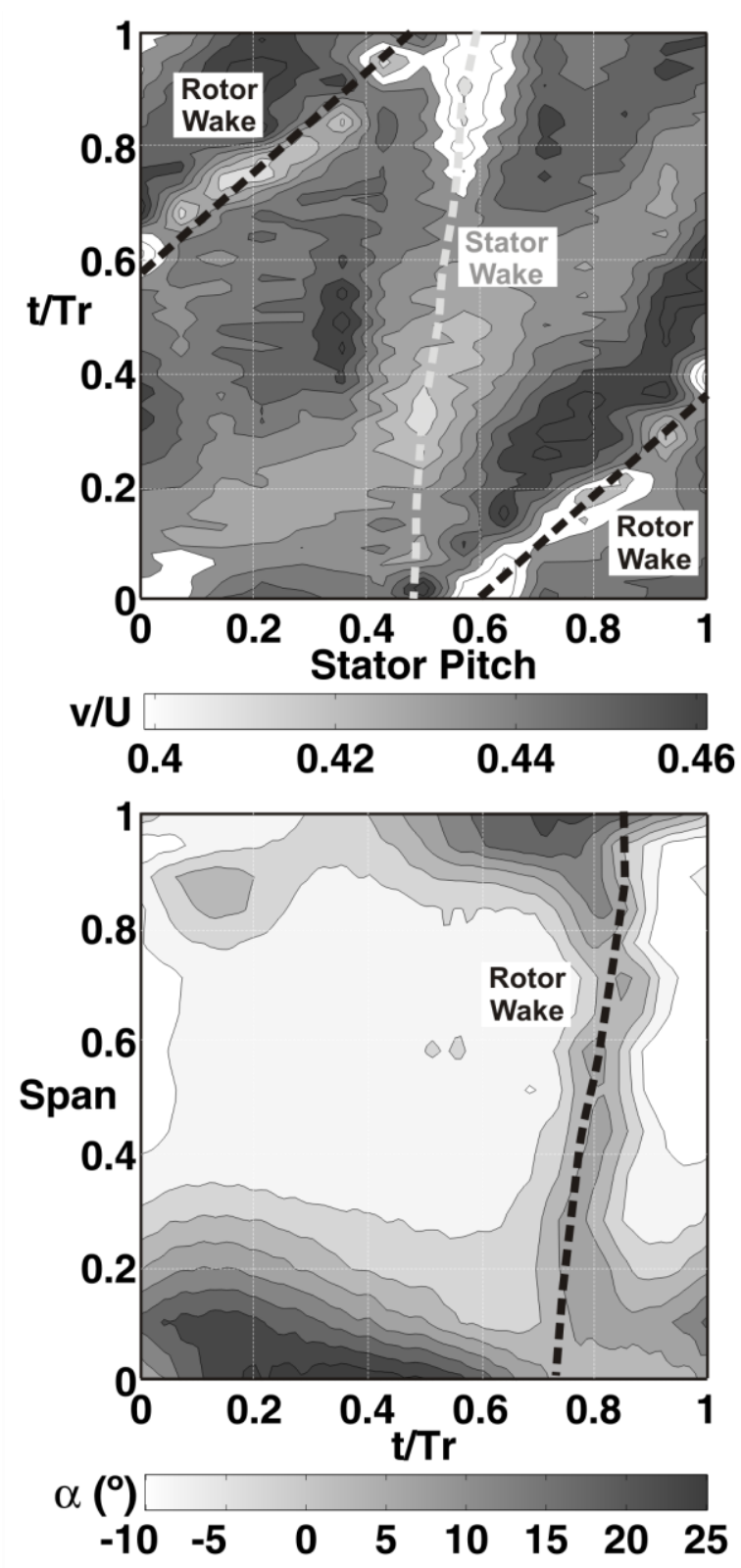

Figure 18. Blade-to-blade maps of both velocity magnitude and flow angle measured with a CTHP.

Complementarily, a set of measurements were conducted in a transverse sector covering the whole span of the machine over one stator pitch. Then, information of the flow was acquired in several circumferential locations for a constant radius (at midspan in the map on top of figure 18); and also in a few radial positions for a particular angular phase (the map in the bottom of the figure). The pressures sensed in the holes are transformed into velocity magnitudes and flow angles as usual. The raw data is additionally ensemble-averaged in order to remove turbulence. Thus, a trigger signal is also employed to enable a correct phase-locked sampling that allows the identification of every rotor phase when ensembleaveraging in the post-processing ([18]). This technique is suitable to obtain temporal evolutions of the velocity field in both radial and circumferential maps, like those represented in figure 18 . 
The map on top shows the circumferential distribution of the velocity field at midspan. The velocity is made non-dimensional with the blade tip velocity as before. The $\mathrm{x}$-axis represents the tangential position, ranging from 0 to 1 as a fraction of a complete stator pitch, while the y-axis represents time non-dimesionalized by $T_{r}$. The drawing reveals a residual wake coming from a stationary stator vane (gray dashed line), since it is fixed to a particular angular position over time. This implies that the stator wakes have not been completely mixed-out when convected throughout the rotor passage. Similarly, the rotor wakes are also identified (black dashed lines) with transversal bands of low velocity running diagonally across the map. Obviously, they are diagonal because they are moving tangentially over time. Nearby the rotor wakes, there is also a transversal band of high velocity that corresponds to the blade-toblade velocity gradient established from the pressure side to the suction side of the blades. The velocity magnitude varies between 0.4 and 0.46 times the blade tip velocity, with the minimum values on the rotor wakes as expected.

Figure 18 is completed with a radial map of the flow angle with respect to the probe axis. In this case, the time is positioned in the abcisa, while the vertical axis represents the normalized span of the machine. Since the measurements were acquired in a particular circunferential position respect to the stator pitch, it is totally representative that no traces of unmixed stator wakes are visible in the map. On the contrary, it is clearly identifiable the presence of a rotor wake (dashed black line) with higher flow angles when $\mathrm{t} / \mathrm{T}_{\mathrm{r}}=$ 0.75. Furthermore, maximum flow angles are associated with the wall annulus boundary layers in both hub and tip regions, while significant uniform flow conditions are observed in the midpassage of the blades. These results, drawn in a spatial-temporal diagram, have been derived from an ensembleaveraging procedure, so the variations of the flow angle are necessary lower (up to $40^{\circ}$ ) than those registered in the instantaneous signals of figure 17. As expected, these measurements have revealed the complexity of the flow patterns downstream of the rotor of an axial turbomachine.

Summarizing, this application has shown the ability of a CTHP, calibrated with the normalization factor $\mathrm{Q}_{\mathrm{N}}$, to measure unsteady two-dimensional flows with significant variations in the flow angle.

\section{CONCLUSIONS}

This paper develops a brand new calibration method for a CTHP working in "non-nulling" mode, which is based on the definition of an improved normalization factor. The new calibration of the probe extends the attainable range of the measurements in 80 degrees with respect to the typical angular ranges derived from traditional calibrations.
Moreover, this new calibration is fulfilled using directly a numerical adjustment of the calibration data, instead of a mathematical curve fitting of the calibration coefficients. Therefore, typical errors associated to manufacturing imperfections or misalignments in the calibration setup are skipped.

It has been confirmed that the angular range of the probe is practically independent of the angular separation of the holes. It is also documented that, with the new calibration, the angular range of the measurements is limited by the angle that is determining the minimum pressure coefficient in the central hole of the probe.

To validate this study, an uncertainty analysis of the new calibration has been carried out. It has been found that the uncertainty is not significantly modified with respect to traditional calibrations, being even lower for certain angular intervals. In any case, global uncertainties in the measurements with this new calibration are perfectly acceptable for all the attainable angular range of the probe.

Additionally, further investigations were undertaken in order to find the optimal construction angle of the probe in terms of uncertainty levels. It was found that the optimal angular distance for the holes is ranged between 50 and 60 degrees. However, those probes designed with separation angles from 45 to 65 degrees are perfectly usuable, since the angular range is practically maintained and the uncertainty maxima can be still assumed.

Finally, a CTHP with an angular distance of 60 degrees was specific-designed to perform experimental measurements downstream of the rotor of an axial turbomachine. Prior to measure, the calibration coefficients derived from the improved normalization factor were determined experimentally. As a result, it was revealed that the attainable angular range of the probe is increased up to $\pm 70^{\circ}$.

A detailed analysis of the jet-wake pattern within the turbomachine passage has confirmed that the probe is capable of capturing the basic flow phenomena, even though high unsteadiness and large variations of the flow angles may be present.

With the new calibration developed in this paper, it is possible to employ a CTHP that enables to measure two-dimensional unsteady flows with variations in the flow angle of up to 140 degrees under acceptable levels of uncertainty.

\section{ACKNOWLEDGEMENTS}

This work was supported by the Research Project "Effect of the volute geometry of centrifugal pumps on the fluidynamic perturbations by means of rotor-stator interaction”, ref. DPI-2006-15034-C02-01, MEC.

\section{NOMENCLATURE}

BL Boundary Layer

BPF Blade Passing Frequency 


$\begin{array}{ll}\text { CTHP } & \text { Cylindrical Three-Hole Probe } \\ \text { FHP } & \text { Five-Hole Probe } \\ \text { LDV } & \text { Laser Doppler Velocimetry } \\ \text { SHP } & \text { Seven-Hole Probe } \\ \text { THP } & \text { Three-Hole Probe } \\ & \\ \text { C } & \text { Central hole } \\ \mathrm{C}_{\alpha} & \text { Angular coefficient } \\ \mathrm{C}_{\mathrm{D}} & \text { Drag coefficient } \\ \mathrm{C}_{\mathrm{p}} & \text { Pressure coefficient } \\ \mathrm{C}_{\mathrm{Po}} & \text { Total pressure coefficient } \\ \mathrm{C}_{\mathrm{Ps}} & \text { Static pressure coefficient } \\ \mathrm{I}_{\alpha} & \text { Angle uncertainty, [deg.] } \\ \mathrm{I}_{\mathrm{C} \alpha} & \text { Angular coefficient uncertainty } \\ \mathrm{I}_{\mathrm{P}} & \text { Pressure uncertainty, }[\mathrm{Pa}] \\ \mathrm{I}_{\mathrm{Ps}} & \text { Static Pressure uncertainty, }[\mathrm{Pa}] \\ \mathrm{I}_{\mathrm{Q}} & \text { Normalization coefficient uncertainty } \\ \mathrm{I}_{\mathrm{QN}} & \text { New normalization coefficient uncertainty } \\ \mathrm{I}_{\mathrm{v}} & \text { Velocity uncertainty, [m/s] } \\ \mathrm{L} & \text { Left hole } \\ \mathrm{P} & \text { Pressure, [Pa] } \\ \mathrm{P}_{\mathrm{C}} & \text { Central hole pressure, [Pa] } \\ \mathrm{P}_{\mathrm{d}} & \text { Dynamic pressure, [Pa] } \\ \mathrm{P}_{\mathrm{L}} & \text { Left hole pressure, [Pa] } \\ \mathrm{P}_{0} & \text { Total pressure, [Pa] } \\ \mathrm{P}_{\mathrm{R}} & \text { Right hole pressure, [Pa] } \\ \mathrm{P}_{\mathrm{S}} & \text { Static pressure, [Pa] } \\ \mathrm{Q} & \text { Normalization coefficient } \\ \mathrm{Q}_{\mathrm{N}} & \text { New normalization coefficient } \\ \mathrm{R} & \text { Right hole } \\ \mathrm{Re} & \text { Reynolds number } \\ \mathrm{St} & \text { Strouhal number } \\ \mathrm{t} & \text { Time, [s] } \\ \mathrm{T}_{\mathrm{r}} & \text { Rotor blade passing period, }[\mathrm{s}] \\ \mathrm{U} & \text { Rotor tip speed, [m/s] } \\ \mathrm{v} & \text { Flow velocity, [m/s] } \\ & \end{array}$

\section{Greek letters}

$\begin{array}{ll}\alpha & \text { Flow angle (yaw angle), [deg.] } \\ \delta & \text { Construction angle of the probe, [deg.] } \\ \theta & \text { Angle around the cylinder, [deg.] } \\ \theta_{\mathrm{S}} & \text { Separation angle, [deg.] }\end{array}$

\section{REFERENCES}

[1] E. Blanco, R. Ballesteros, C. Santolaria, Angular Range and Uncertainty Analysis of Nonorthogonal Crossed Hot Wire Probes. Journal of Fluids Engineering 120 (1998) 90-94.

[2] T.J. Dudziniski, L.N. Krause, Effect of Inlet Geometry on Flow-Angle Characteristics of
Miniature Total-Pressure Tubes. NASA TN D6406 (1971).

[3] J.R. Erwin, Experimental Techniques. Section D of Aerodynamics of Turbines and Compressors: Princeton University Press (1964).

[4] T.J. Dudziniski, L.N. Krause, Flow-Direction Measurement with Fixed-Position Probes. NASA TM X-1904 (1969).

[5] W. Wuest, Measurement of Flow Speed and Flow Direction by Aerodynamic Probes and Vanes. AGARD Conference Proceedings 32 (1967) 373426.

[6] W.E. Lewis, Fixed-Direction Probes for Aerodynamic Measurements. Proc. Inst. Mech. Eng. 180 (1966) 141-152.

[7] D.W. Bryer, R.C. Pankhurst, Pressure-Probe Methods for Determining Wind Speed and Flow Direction. National Physical Laboratory, ISBN: 011-480012X (1971).

[8] G.G. Zilliac, Calibration of Seven-Hole Pressure Probes for Use in Fluid Flows with Large Angularity. NASA TM 102200 (1989).

[9] A.L. Treaster, A.M. Yocum, The Calibration and Application of Five-Hole Probes. ISA Transactions 18 (1979) 23-34.

[10] K.N. Everett, A.A. Gerner, D.A. Durston, SevenHole Probe for High Angle Flow Measurement: Theory and Calibration. AIAA J. 21 (1983) 992998.

[11]R.W. Gallington, Measurement of Very Large Flow Angles with Non-Nulling Seven-Hole Probes. Aeronautics Digest: USAF Academy.

[12] S.O. Kjelgaard, Theoretical Derivation and Calibration Technique of a Hemispherical-Tipped Five-Hole Probe. NASA TM 4047 (1988).

[13]D. Sumner, A Comparison of Data-Reduction Methods for a Seven-Hole Probe. Journal of Fluids Engineering 124 (2002) 523-527.

[14] G.L. Morrison, M.T. Schobeiri, K.R. Pappu, FiveHole Pressure Probe Analysis Technique. Flow Measurement and Instrumentation 9 (1998) 153158.

[15]F.M. White, Fluid Mechanics. McGraw-Hill, ISBN: 84-481-4076-1 (2003).

[16]P.D. Weidman, Wake Transition and Blockage Effects on Cylinder Base Pressures. Ph.D. Thesis: California Institute of Technology (1968).

[17]S.J. Kline, F.A. McClintock, Describing Uncertainties in Single Sample Experiments. Mechanical Engineering (1953) 3-8.

[18]Lakshminarayana, B., Techniques for Aerodynamic and Turbulence Measurements in Turbomachinery Rotors. ASME Journal of Engineering. and Power 103 (1981) 374-392. 
This document is a pre-print version of the scientific paper published by Elsevier. It has been released by the authors to fulfill all the publisher requirements established for Article Sharing: https://www.elsevier.com/about/policies/sharing

\section{(9) $\Theta \Theta \Theta$}

(C) 2019. This manuscript version is made available under the Creative Commons Attribution-NonCommercial-NoDerivatives 4.0 International License (CC-BY-NC-ND 4.0 license) http://creativecommons.org/licenses/by-nc-nd/4.0/ 